\title{
Beneficios del uso de cal hidratada en mezclas asfálticas: Revisión del estado del arte
}

\author{
Benefits of adding hydrated lime to asphalt mixtures: A review
}

\section{Karina Kikut Cruz}

LanammeUCR, Universidad de Costa Rica,

Costa Rica

karina.kikut@ucr.ac.cr

\section{M.Sc. Alejandra Baldi}

LanammeUCR, Universidad de Costa Rica,

Costa Rica

alejandra.baldi@ucr.ac.cr

\section{M.Sc. Ana Luisa Elizondo Salas}

LanammeUCR, Universidad de Costa Rica,

Costa Rica

ana.elizondosalas@ucr.ac.cr

Fecha de recepción: 30 de abril de 2020 / Fecha de aprobación: 01 de julio de 2020

\section{RESUMEN}

La cal hidratada es un material utilizado normalmente en las mezclas asfálticas, dado que les brinda propiedades únicas y les permite alargar su vida útil. En el presente trabajo se analizaron diversos artículos con el objetivo de comprender los métodos por medio de los cuales se agrega la cal hidratada, la cuantificación del compuesto y la mejora que éste aporta ante diversos factores adversos que acometen a las mezclas asfálticas. Se encontró que los porcentajes usuales en los que se utiliza este aditivo corresponden a un rango entre $1 \%-2 \%$ en peso de los agregados. Asimismo, utilizar cal como un aditivo en húmedo permite mejoras tanto en la dureza del asfalto como en la resistencia contra la humedad, de asfalto. Se concluye que la cantidad de cal hidratada que se debe agregar corresponde al 1,5\% en masa de agregados debido a que, en la mayoría de estudios, con esta cantidad se obtuvo las mejores propiedades del asfalto.

\begin{abstract}
Hydrated lime is used as an additive for asphalt mixtures, since it enhances the properties of the material in a unique way, enabling a longer service life. In this study, several scientific articles were analyzed in order to understand some of the methods employed of adding hydrated lime to the asphalt mixtures, as well as the optimum amount of hydrated lime that will significantly improve the material's response towards diverse factors. It was found that the researchers usually add hydrated lime in percentages ranging between 1\%-2\% in weight of aggregates. Also, in the case of asphalt modified with hydrated lime, the material is stiffer and more resistant to moisture damage. Finally, after this literature review it is concluded that adding 1,5\% of hydrated lime the most promising properties were obtained in most of the studies considered.
\end{abstract}

Keywords: asphalt, hydrated lime, moisture damage, asphalt matrix.

Palabras clave: asfaltos, cal hidratada, daño por humedad, matriz asfáltica. 


\section{INTRODUCCIÓN}

La cal hidratada es un compuesto que se ha estudiado ampliamente en los últimos años como un agregado en la matriz asfáltica por los beneficios que esta le brinda. A este aditivo se le conoce también como filler; y de acuerdo con Little y Petersen (2005), se puede definir filler como "cualquier partícula que se agregue al asfalto con un tamaño menor a los $74 \mu m$ ". Asimismo, los autores detallan que la cal hidratada se añade al asfalto por sus propiedades excepcionales, como el tamaño de partícula que posee, la rugosidad y la energía superficial de la misma, entre otras.

Hay diversas razones por las que se utiliza este material como agregado, una de ellas es por la resistencia a fisura que le agrega al asfalto. Según Rasouli, Kavussi, Qazizadeh y Taghikhani, (2018), la cal hidratada puede mejorar las propiedades de la mezcla asfáltica de tres maneras: mejorando la resistencia al agrietamiento, mejorando la resistencia a la deformación permanente y reduciendo la tasa de rigidez a causa de la oxidación del material.

Asimismo, la cal hidratada es un aditivo de uso común debido a las propiedades que le brinda al asfalto para resistir el daño por humedad; la cual es una de las causas del fallo prematuro de la mezcla asfáltica. Es por ello que a lo largo de los años se ha tratado de implementar pruebas para determinar la sensibilidad del asfalto ante la humedad. Existen estudios que basan esta sensibilidad en parámetros de fractura, energía superficial, coeficientes de difusión y características de adhesión. La razón por la cual se emplea cal hidratada es porque esta aumenta las características de unión adhesiva entre el agregado y el asfalto (Kim, Lutif, Bhasin y Little, 2008).

Debido a que la cal hidratada se ha utilizado ampliamente para mitigar los daños que sufre el asfalto por la humedad, se decidió realizar una investigación bibliográfica para comprender tanto las propiedades de la cal, como los métodos de adición a la matriz asfáltica y las pruebas que se han realizado a la mezcla asfáltica una vez agregado este compuesto.

Asimismo, es importante destacar que el daño por humedad es de los principales problemas que presentan el asfalto en Costa Rica, debido a que este es un país con un clima tropical donde la época lluviosa corresponde a seis meses del año. De igual manera, Costa Rica posee un único asfalto por lo que este artículo pretende brindar una revisión literaria del uso de cal para mitigar dichos problemas; desde cómo modificarlo, las cantidades óptimas y las mejoras en las propiedades mecánicas que se deben esperar.
El objetivo de este estudio es lograr una noción integral de la técnica de aplicación de cal en las mezclas asfálticas y mástic asfáltico, para poder ser aplicada en Costa Rica.

\section{DESARROLLO Y DISCUSIÓN}

\section{Contenido óptimo de cal hidratada en mezclas asfálticas}

Zamora y Mora (2019), utilizaron briquetas con una masa de $1 \mathrm{~kg}$ cada una; de la cual 47,45 g correspondió al filler y este no poseía cal hidratada. Posteriormente, se sustituyó el filler al $100 \%$ por cal hidratada y al $50 \%$; y se les realizaron pruebas de tracción seca, tracción húmeda y desgaste. A partir de las pruebas realizadas los autores determinaron que el porcentaje óptimo de sustitución del filler por cal hidratada fue de 2,387\% del peso total de la mezcla.

Por otro lado, Bastidas, De Carvalho, y Lucena, (2015) realizaron un estudio con muestras asfálticas modificadas con: $0 \%$ cal, $10 \%$ cal, $15 \%$ cal y $20 \%$ cal. Las mezclas con mayores porcentajes de cal tendieron a sedimentarse y producir una mezcla heterogénea. A partir del estudio los autores proponen utilizar 0,96\% de cal hidratada y 3,84\% de asfalto puro; estos datos surgen a partir de que el contenido de asfalto efectivo se reduce en un $20 \%$ por la adición de cal. Este $20 \%$ se traduce en un $0,3 \%$ del contenido óptimo de un asfalto en un concreto denso.

Lesueur, Petit, y Ritter (2013), exponen que la cantidad típica de cal que se agrega a la mezcla asfáltica está en el rango de $1 \%$ a $1,5 \%$ en masa de agregados secos. Si se utilizara la cal como aglutinante típico, su contenido debe ser del $5 \%$ en masa con base en agregados secos, este porcentaje corresponde al 20\%-30\% en masa o a $10-15 \%$ en volumen de cal hidratada. Asimismo, la Asociación Europea de Cal (European Lime Association), realizó en el año 2011, una tabla comparativa de la cantidad de cal hidratada que se agrega al asfalto por país, así como el método de adición, y partir de esto se determina que el rango de porcentajes de cal hidratada agregada ronda el $1 \%$ a 3,5\% y esta se agrega pura o mezclada con el filler (EuLa, 2011).

Por su parte, Han, Dong, Yin, Liu, y Liu (2020), se enfocaron en hallar la cantidad óptima de cal hidratada, así como su grado de fineza idóneo para la mezcla asfáltica. A partir de este estudio se obtuvo que el contenido óptimo de cal corresponde al 10\%. También se descubrió que, el contenido de cal se debe controlar para asegurarlo entre 3\% y $10 \%$, para mantener las propiedades reológicas adecuadas del asfalto. 
En cambio, Little y Epps realizaron un estudio sobre el uso de cal hidratada, desde su historia hasta los métodos de adición a la matriz asfáltica. Los autores explicaron que la cal seca se puede añadir como agregados secos o húmedos; y los estudios que compilan emplean entre 1\%-3\% de cal hidratada (National Lime Association, 2006).

De acuerdo con el estudio bibliográfico realizado, se concluye que, si bien es cierto que hay diversos porcentajes en los que se agrega la cal hidratada al asfalto, los más utilizados se encuentran entre $1 \%$ y $2 \%$ del peso en agregados.

\section{Uso de cal hidratada para mejorar el desempeño del asfalto y de la mezcla asfáltica}

Kim et al. (2008), analizaron mezclas asfálticas a las que se les agregó 1\% cal hidratada variando la concentración de cal, la cual se agregó tipo lodo. Estas concentraciones fueron 0,$33 ; 0,22$ y 0,13 porciento. A todas se les realizaron pruebas de daño por humedad, susceptibilidad, fractura y energía libre superficial, entre otras. Los resultados se reportan en conjunto, por lo que no se reporta el porcentaje óptimo de cal hidratada que se debe agregar; sin embargo, con el 1\% sí se observa una mejora de las propiedades del asfalto.

En el caso del estudio de Kollaros, Kalaitzaki, y Athanasopoulou (2017), este se centró en la investigación de fillers para mezclas asfálticas, así como su dosificación. Los fillers que se probaron fueron agregados pasando el tamiz No.200 y cal. El contenido de filler se varió en $2 \%, 4 \%, 6 \%$ y $8 \%$ y se realizaron pruebas de estabilidad, vacíos y deformación. La adición de cal significó mejoras en todas las pruebas mencionadas anteriormente, la máxima estabilidad se obtuvo con el $4 \%$ de filler, mientras que la deformación, con el $2 \%$.

Rasouli y colaboradores (2018) utilizaron especímenes con porcentajes entre el $1 \%$ y $2 \%$ de contenido en masa de cal hidratada. Les realizaron pruebas de fatiga y determinaron que con la modificación se aumentó la dureza de la mezcla asfáltica; así como que hubo una mejora de las propiedades elásticas de la mezcla. Los autores descubrieron que al agregar un porcentaje mayor al $1,5 \%$ de cal hidratada se podría conseguir una disminución gradual de flexibilidad de la mezcla y un aumento excesivo de la dureza.

Moon, Falchetto, Wang, Riccardi y Wistuba (2017), analizaron el efecto de adicionar cal hidratada a la mezcla asfáltica como parte del filler, variando los porcentajes añadidos de la siguiente forma: 5\%, 10\% y $20 \%$ en volumen de agregado fino. Analizaron el desempeño de las mezclas a temperatura baja $\left(10^{\circ} \mathrm{C}\right.$ y $\left.-6^{\circ} \mathrm{C}\right)$ y alta $\left(28^{\circ} \mathrm{C}\right.$ y $\left.70^{\circ} \mathrm{C}\right)$. Los autores encontraron que a bajas temperaturas las propiedades de la mezcla no se ven afectadas significativamente. Por su parte, a altas temperaturas hubo una mejora en las propiedades de la mezcla con ambos tipos de filler.

Otras pruebas diferentes a las discutidas anteriormente las realizó Gutiérrez (2017), al agregar cal hidratada al asfalto en el agregado fino y realizando tres pruebas a las muestras; estas pruebas fueron: equivalente de arena, determinación del peso específico y análisis granulométrico. Las proporciones en las que se agregó la cal hidratada correspondieron a 1\%, 1,5\% y $2 \%$. La que brindó los mejores resultados fue la de 1,5\%, dado que con esta cantidad se obtuvo la mayor estabilidad de la mezcla.

Por otro lado, es común añadir cal directamente al asfalto para evaluar su respuesta ante el envejecimiento químico, el cual es un factor importante que acomete al asfalto y reduce su vida útil. Es también un factor difícil de cuantificar para conocer su avance. Lo anterior, se debe a la dificultad en la toma de muestras del asfalto envejecido, debido a que se deben extraer los primeros centímetros o milímetros de la capa asfáltica, que en ocasiones se mezclan con capas inferiores que no son representativas del envejecimiento químico (EuLA, 2011).

Entonces, se ha probado el efecto de la modificación con cal hidratada y cal hidratada dolomítica sobre el índice de envejecimiento obtenido a $60^{\circ} \mathrm{C}$ (EuLA, 2011). Entre los resultados obtenidos, se observa la disminución de la susceptibilidad del envejecimiento químico del asfalto mediante un aumento paulatino de la viscosidad. Asimismo, se da una disminución de la formación de carbonilos en los asfaltos modificados; este efecto surge a temperaturas mayores a los $88^{\circ} \mathrm{C}$. Además, con $10 \%$ de uso de cal hidratada se neutralizaron la mayoría de los ácidos en el asfalto (EuLA, 2011).

Adicionalmente, el experimento elaborado por Aragão, Lee, Kim y Karki (2010) se centró en analizar los efectos de la cal hidratada en el asfalto, para mejorar la resistencia al fisuramiento. La cantidad de cal hidratada se varió en las siguientes proporciones: $0,5 \%, 1,0 \%, 1,5 \%, 2,0 \%$ y 3,0\% en peso de agregado seco. Las pruebas realizadas corresponden a módulo dinámico, resistencia ante la fatiga, y deformación permanente. Una vez más, se observó una mejora en la dureza de la mezcla asfáltica, así como una mejora en la resistencia ante la fatiga de las muestras. Al agregar contenidos mayores al 2\% de cal hidratada a la mezcla no se observó una mejora adicional en sus propiedades. 
Lesueur et al. (2013), realizaron una recopilación bibliográfica de la modificación de asfalto con cal hidratada; concluyendo que este aditivo incrementa la durabilidad del asfalto. Este incremento de la durabilidad se logra a partir de la mejora que proporciona la cal hidratada a las propiedades como la resistencia ante el daño por humedad, la resistencia ante el envejecimiento químico, y las propiedades mecánicas (módulo de resistencia, fuerza y fatiga, entre otras). Han et al. (2020), por su parte, realizaron pruebas de energía libre superficial, las propiedades técnicas de la cal y las propiedades mecánicas al asfalto modificado. Se obtuvo que, al aumentar la fineza de la cal, se aumenta también la adhesión del asfalto; y que al agregar cal hidratada aumenta la viscosidad y dureza del asfalto.

En el 2005, Little y Petersen (2005) realizaron un estudio que tuvo como enfoque comparar la adición de cal y cal hidratada en la reología de la matriz asfáltica. La cal hidratada se agregó como filler en una proporción del $20 \%$ y se le realizaron pruebas mecánicas. Cabe destacar que el $20 \%$ en masa de cal hidratada mencionado anteriormente corresponde al $1 \%$ típico en mezclas de pavimentos. Se descubrió que cal le brinda al asfalto características importantes, una de ellas corresponde a la dureza, que le otorga resistencia a la mezcla asfáltica ante posibles fracturas o fisuras. Cabe destacar que mostró mejores características el asfalto modificado con cal hidratada que el que se modificó con cal.

\section{Uso de la cal hidratada para mitigar el daño por humedad en mezclas asfálticas}

Como se mencionó anteriormente, la cal hidratada brinda diversas propiedades al asfalto, de forma tal que lo convierte en un material más resistente ante factores adversos, como lo es la humedad. Asimismo, posteriormente se ahondará en los avances que se han realizado en el uso de la cal hidratada para mitigar el daño por humedad en Costa Rica.

El daño por humedad consiste en la infiltración de humedad o agua en el asfalto provocando la separación entre este y los agregados. Caro, Masad, Bhasin y Little (2008), lo definen como: "la degradación de las propiedades mecánicas de un material debida a la presencia de humedad en estado líquido o de vapor." De acuerdo con Kim et al. (2008), este tipo de daño genera diversas señales en el material, como lo es el agrietamiento y la deformación permanente del asfalto. Existen algunos mecanismos para estudiar y comprender el daño por humedad; Caro et al. (2008), exponen que estos mecanismos se basan en dos pasos: transporte de humedad y la respuesta del sistema. El primero es para comprender la forma en la que la humedad llega al asfalto, y el segundo coresponde a cómo se modificó la estructura interna del material. Algunas manifestaciones de la humedad en el asfalto corresponden al agrietamiento, desnudamiento, desmoronamiento, y erosión hidráulica de la mezcla asfáltica (Caro et al., 2008).

Kim et al. (2008), inician su investigación ahondando en el daño prematuro del asfalto a causa de la humedad y el surgimiento de técnicas para reducir este tipo de daño. Una de las técnicas más utilizadas para reducir el daño por humedad en mezclas asfálticas consiste en agregar cal hidratada, pues es conocido que ésta mejora la compatibilidad adhesiva entre el agregado y el asfalto. Hay diversas formas de agregar la cal hidratada al asfalto, por ejemplo: agregándola a los agregados secos o a los agregados húmedos, entre otros. La razón por la cual la cal hidratada reduce el daño por humedad en el asfalto es que ésta agrega iones de calcio en la superficie del agregado, logrando que tanto los agregados como el asfalto tengan mejor afinidad para resistir el daño por humedad. La cal logra reaccionar con el agua y el dióxido de carbono del aire formando carbonato de calcio que resulta en una superficie dura del mineral y mejorando la adhesión entre los agregados y el asfalto (Han et al., 2020).

Kim et al. (2008), realizaron dos pruebas de laboratorio las cuales consistieron en evaluar el daño por humedad, la susceptibilidad del asfalto, la medición de las propiedades del material y la posibilidad de fractura de este. Como principal conclusión reportan que las mezclas que contenían cal hidratada tuvieron mejor rendimiento en los siguientes aspectos: 1) aumento de la rigidez, 2) aumento de la fuerza y la dureza, que generan mejor resistencia ante la degradación por humedad, y 3) mejor adherencia entre el asfalto y el agregado, que produce mejor resistencia ante las fisuras.

Mouillet, Séjourné, Delmotte, Ritter, y Lesueur (2014), reconocen el uso de la cal hidratada para mitigar los efectos de la humedad en el asfalto, así como para la reducción del envejecimiento químico del asfalto. Además, se encontró que agregar cal aumenta la dureza del asfalto que impacta directamente las propiedades mecánicas del material, esto al punto de que las agencias norteamericanas estiman que agregar cal hidratada a la mezcla asfáltica aumenta la durabilidad del material entre 2 y 10 años (un incremento del 20-50\%).

Khattak y Kyatham, realizaron dos estudios en el mismo año. En el primero evaluaron la susceptibilidad tanto de la matriz asfáltica como de la mezcla asfáltica ante la humedad, modificando ambas con cal hidratada. El aglutinante asfáltico se modificó con $20 \%$ de cal hidratada; este no se utilizó como 
filler. La matriz asfáltica se utilizó con $10 \%$ en contenido de aglutinante asfáltico y el agregado fino que pasara por el tamiz No.8. Utilizando el método de área superficial, el aglutinante correspondió entre el 18\%-20\% en peso de la matriz asfáltica. Se obtuvo una respuesta viscoelástica positiva al haber modificado la mezcla con cal hidratada, es decir, un aumento en el valor del módulo dinámico, G* (Khattak y Kyatham, 2008a). En el segundo estudio, se enfocaron en el agregado fino debido a que es más susceptible al daño por humedad. Se modificó el mismo con cal hidratada, en 20\% para el aglutinante. La matriz asfáltica se compuso de la misma forma que la explicada anteriormente. A partir de diversas pruebas, se da una mejora en la resistencia de la matriz asfáltica que la hace menos susceptible ante el daño por humedad (Khattak y Kyatham, 2008b).

Los autores Little y Epps determinan que la cal hidratada reduce la sensibilidad del asfalto ante la humedad y mejora la adhesión entre el agregado y el asfalto. Asimismo, esta sustancia reduce el envejecimiento del material por oxidación y aumenta la resistencia de este ante la fatiga. Un estudio demostró que al agregar cal hidratada se puede dar un ahorro de $\$ 20 /$ ton de mezcla asfáltica, y puede alargar la vida útil del material en un 38\% (National Lime Association, 2006).

Es importante mencionar los métodos para cuantificar el daño por humedad, algunos de estos son: HWTD (Hamburg Wheel Tracking Device por sus siglas en inglés), ITSR (Indirect tensile strength ratio por sus siglas en inglés), Duriez, Cantabro, SATS (Saturation ageing tensile stiffness por sus siglas en inglés), y Lottman, entre otros. Cabe destacar que aún no hay estudios que determinen cuál método a utilizar es el más adecuado para evaluar el daño por humedad. Al hacerse la comparación de los distintos agregados (incluyendo cal hidratada) y diferentes métodos de prueba se ha podido observar que en la prueba Lottman la cal hidratada tiene un mejor desempeño que otros aditivos. También en la prueba HWTD se demuestra que la cal hidratada es más eficiente al mejorar la resistencia del asfalto para el daño por humedad (EuLA, 2011).

En la totalidad de los estudios consultados se determina que agregar cal hidratada tiene un beneficio claro en las propiedades del asfalto, aumentando su dureza, resistencia ante la humedad y mejora las características de la matriz asfáltica, en general.

\section{Uso de cal hidratada en la mezcla asfáltica en Costa Rica}

En Costa Rica se han realizado investigaciones para la incorporación de cal hidratada como agregado en la matriz asfáltica para mitigar el daño por humedad. Jiménez (2015), realizó la modificación del ligante asfáltico con nanocal, usándose este tamaño nanométrico para lograr una mejor interacción entre las partículas de ligante y las de cal. A partir de esta investigación se concluyó que no hay una mejora en el grado de desempeño del asfalto (según la metodología Superpave) al comparar la utilización de nanocal o cal convencional. Asimismo, de acuerdo con los parámetros de resistencia retenida y pérdida de adherencia, los asfaltos modificados con cal mejoraron desde $5 \%$ hasta $11 \%$.

De igual forma, Vargas (2016), evaluó los efectos de agregar cal y nanocal a las mezclas asfálticas en caliente con tamaños nominales de $9,5 \mathrm{~mm}$ y $12,5 \mathrm{~mm}$. A las muestras se le realizaron tres ensayos los cuales fueron: Tensión Diametral, Rueda de Hamburgo y Módulo Dinámico. De la investigación se obtuvo que al agregar cal y nanocal se reducen los porcentajes de asfalto óptimo requerido; por lo que este se traduce en una reducción de los costos de producción. La muestra que presentó los resultados más favorables correspondió al asfalto de 12,5 mm modificado con cal; el cual posee mejor resistencia ante el daño por humedad.

En el 2016 se preparó un informe de daño por humedad en mezclas asfálticas por parte del Laboratorio Nacional de Materiales y Modelos Estructurales, su objetivo correspondió en la preparación de una metodología para lograr cuantificar el daño por humedad en mezclas asfálticas para las condiciones de Costa Rica. Como hallazgos del informe se obtuvo una mejora de la resistencia ante el daño por humedad de las mezclas modificadas con cal. También, se encontró que los modificadores que añadieron a las mezclas asfálticas, como el polímero SBS o la cal, afectan las propiedades volumétricas de la mezcla (LanammeUCR, 2016).

En el 2017 se realizó otro informe para el daño por humedad, cuyo objetivo correspondió a evaluar de manera exhaustiva la susceptibilidad al daño por humedad, de diversas mezclas asfálticas, a través de un análisis por componentes y conglomerados. A partir de este informe, se concluyó que al agregar cal a la mezcla asfáltica hay una ganancia de resistencia al daño por humedad. También se destacó que, al modificar las mezclas asfálticas con el polímero SBS (poliestirenobutadieno-estireno) y agente antidesnudante líquido (ADL), se mejora el desempeño del material ante el daño por humedad y agrietamiento por fatiga (LanammeUCR, 2017). 
Por otro lado, en el 2018 se realizó una investigación similar en el Lanamme, la cual concluyó que el parámetro actual definido, utilizado como criterio de aceptación ante el daño por humedad no discrimina si la mezcla es susceptible o no ante dicho problema; lo cual corresponde a la realidad de los pavimentos en Costa Rica. De igual manera, los investigadores concluyeron que, al adicionar cal a la mezcla asfáltica, se observa una mejora en la resistencia del material ante el daño por humedad; en especial cuando la mezcla se sometió a un nivel mayor de daño (LanammeUCR, 2018).

\section{Métodos de Cuantificación de la Cal Hidratada en la Matriz Asfáltica}

Muchos de los estudios consultados se basan en la cantidad usual en que se agrega cal hidratada, dado que ha sido una técnica muy estudiada a lo largo de los años. Sin embargo, a falta de cuantificar la cantidad de cal adicionada podría experimentarse problemas de sedimentación. De igual forma, se evidenció que, si bien la cal hidratada brinda beneficios al asfalto, lo hace cuando se agrega en cierta proporción; más allá de la proporción indicada se desperdicia el compuesto y no hay un beneficio adicional en sus propiedades.

Uno de los métodos de cuantificación es el método estadounidense, que emplea la espectroscopía infrarroja con transformada de Fourier (FTIR, por sus siglas en inglés) en el cálculo del contenido de cal hidratada en asfaltos. La señal es sencilla de hallar en el espectro y se presenta en longitudes de onda de 1390-1400 $\mathrm{cm}^{-1}$ (Mouillet et al., 2014).

Otro método de cuantificación corresponde al alemán; este método consiste en tres pruebas: la pureza de la cal hidratada, el contenido de cal hidratada en la mezcla como filler y lo que se recupera de la cal en el filler proveniente de una mezcla asfáltica. Esta prueba se basa en la titulación con ácido clorhídrico de una muestra en suspensión con el producto de interés. El filler se recolecta luego de la extracción del asfalto de la mezcla. La suspensión por titular se obtiene disolviendo $1 \mathrm{~g}$ del filler recolectado en $150 \mathrm{~mL}$ de agua, $10 \mathrm{~mL}$ isobutanol y $5 \mathrm{~mL}$ de una solución surfactante. Este método se aplicó a muestras asfálticas 1,5 años después de haber sido colocadas. Emplearon 4 muestras de las cuales 2 contenían cal hidratada en un $25 \%$ en masa y la cantidad de cal hidratada posterior a la prueba fue mayor para ambas muestras. Los autores determinaron que no toda la cal hidratada agregada se recuperó debido a las reacciones entre los agregados y la cal (Mouillet et al., 2014).

\section{CONCLUSIONES}

La investigación bibliográfica realizada sugiere que, al adicionar cal hidratada a la matriz asfáltica, hay una mejora en las propiedades de la mezcla en cuanto a la dureza y la resistencia ante la fatiga, oxidación, envejecimiento químico y daño por humedad. Asimismo, se observan cambios en las propiedades viscoelásticas del asfalto, cuando la cal hidratada se mezcla, mejorando su resistencia a la deformación permanente.

Por otro lado, con base en los estudios consultados, se infiere que la cantidad óptima de cal en la mezcla asfáltica corresponde al $1,5 \%$ en peso de agregados para obtener mejores propiedades y que el material resista a la humedad.

Es importante recalcar que los resultados obtenidos y los beneficios que se hallaron tras el uso de la cal, están ligados al objetivo de cada estudio analizado. Es decir, aquellas investigaciones enfocadas en analizar el efecto de la cal sobre cierta propiedad de la mezcla o del asfalto modificado con este material, presentarán resultados relacionados con dicha propiedad en específico. Es por ello, que se recomienda discreción por parte de los lectores, dado que, a pesar de que este aditivo es en efecto beneficioso para el pavimento, podría percibirse como una solución a todos los deterioros que ocurren en la carretera.

Se recomienda ahondar en las investigaciones sobre el uso de este agregado en la matriz asfáltica en Costa Rica, para determinar su viabilidad y aplicabilidad en las carreteras del país. 


\section{REFERENCIAS}

Aragão, F., Lee, J., Kim, Y., y Karki, P. (2010). Material-Specific Effects of Hydrated Lime on the Properties and Performance Behavior of Asphalt Mixtures and Asphaltic Pavements. Construction and Building Materials, 24(4), 538-544. doi: 10.1016/j. conbuildmat.2009.10.005

Bastidas, J., De Carvalho, J., y Lucena, L. (2015). Optimización del contenido de asfalto modificado con cal hidratada en mezclas asfálticas densas. Revista Asfaltos y Pavimentos, 31, 55-64.

Caro, S., Masad, E., Bhasin, A., y Little, D. N. (2008). Moisture susceptibility of asphalt mixtures, Part 1: mechanisms. International Journal of Pavement Engineering, 9(2), 81-98. doi: 10.1080/10298430701792128

EuLA (2011). Hydrated Lime: A Proven Additive for Durable Asphalt Pavements Critical Literature Review. Recuperado de: https:// www.eula.eu/hydrated-lime-a-proven-additive-for-durable-asphalt-pavements-critical-literature-review-paper/

Gutiérrez, L. (2017). Diseño de asfalto en caliente mejorado con el uso de cal hidratada en la Av. Camino Dibos, ICA, 2017. (Tesis de grado). Universidad César Vallejo, Lima.

Han, S., Dong, S., Yin, Y., Liu, M., y Liu, Y. (2020). Study on the effect of hydrated lime content and fineness on asphalt properties. Construction and Building Materials, 244, 1-9. doi: 10.1016/j.conbuildmat.2020.118379

Jiménez, M. (2015). Análisis del efecto de nano partículas de cal hidratada en el desempeño del ligante asfáltico. (Tesis de grado). Universidad de Costa Rica, San José.

Khattak, M., y Kyatham, V. (2008a). Mechanistic Characteristics of Moisture Damaged Asphalt Matrix and Hot Mix Asphalt Mixtures. Airfield and Highway Pavements 2008, Washington.

Khattak, M., y Kyatham, V. (2008b). Viscoelastic Behavior of Hydrated Lime-Modified Asphalt Matrix and Hot-Mix Asphalt Under Moisture Damage Conditions. Journal of the Transportation Research Board, 2057(1), 64-74. doi: 10.3141/2057-08

Kim, Y., Lutif, S., Bhasin, A., y Little, D. (2008). Evaluation of Moisture Damage Mechanisms and Effects of Hydrated Lime in Asphalt Mixtures through Measurements of Mixture Component Properties and Performance Testing. Journal of Materials in Civil Engineering, 20(10), 659-667. doi: 10.1061/(ASCE)0899-1561(2008)20:10(659)

Kollaros, G., Kalaitzaki, E., y Athanasopoulou, A. (2017). Using Hydrated Lime in Hot Mix Asphalt Mixtures in Road Construction. American Journal of Engineering Research (AJER), 6(7), 261-266.

Lesueur, D., Petit, J., y Ritter, H. (2013). The mechanisms of hydrated lime modification of asphalt mixtures: A state-of-the-art review. Road Materials and Pavement Design, 14(1), 1-16. doi: 10.1080/14680629.2012.743669

Little, D., y Petersen, C. (2005). Unique Effects of Hydrated Lime Filler on the Performance- Related Properties of Asphalt Cements: Physical and Chemical Interactions Revisited. Journal of Materials in Civil Engineering, 17(2), 207-218. doi: 10.1061/ (ASCE)0899-1561(2005)17:2(207)

Moon, K., Falchetto, A., Wang, D., Riccardi, C., y Wistuba, M. (2017). Mechanical Performance of Asphalt Mortar Containing Hydrated Lime and EAFSS at Low and High Temperatures. Materials, 10(7), 1-20. doi: 10.3390/ma10070743

Mouillet, V., Séjourné, D., Delmotte, V., Ritter, H., y Lesueur, D. (2014). Method of quantification of hydrated lime in asphalt mixtures. Construction and Building Materials, 68, 348-354. doi: 10.1016/j.conbuildmat.2014.06.063

National Lime Association (2006). The Benefits of Hydrated Lime in Hot Mix Asphalt. Recuperado de: https://scholarworks.unr. edu/handle/11714/4927

Rasouli, A., Kavussi, A., Qazizadeh, M., y Taghikhani, A. (2018). Evaluating the effect of laboratory aging on fatigue behavior of asphalt mixtures containing hydrated lime. Construction and Building Materials, 164, 655-662. doi: 10.1016/j. conbuildmat.2018.01.003 
LanammeUCR (2016). Daño por humedad en mezclas asfálticas (Informe LM-PI-UMP-044-R3). Recuperado de: https://www. lanamme.ucr.ac.cr/repositorio/handle/50625112500/991

LanammeUCR (2017). Daño por humedad en mezclas asfálticas (Informe LM-PI-UMP-044-R4). Recuperado de: https://www. lanamme.ucr.ac.cr/repositorio/handle/50625112500/977

LanammeUCR (2018). Daño por humedad en mezclas asfálticas (Informe LM-PI-UMP-044-R5). Recuperado de: https://www. lanamme.ucr.ac.cr/repositorio/handle/50625112500/1710

Vargas, M. (2016). Efecto en la resistencia al daño por humedad de la mezcla asfáltica en caliente al añadir cal y nano cal hidratada (Tesis de grado). Universidad de Costa Rica, San José.

Zamora, F., y Mora, E. (2019). Influencia de la cal hidratada en mezclas asfálticas drenantes (Tesis de grado). Universidad Católica de Colombia, Bogotá. 